\title{
Clinical significance of $B R A F$ V600E mutation in 154 patients with thyroid nodules
}

\author{
LINGYING YU, LIZHEN MA, QIAOFENG TU, YI ZHANG, YUEMING CHEN, DAOJUN YU and SHAOYU YANG \\ Department of Endocrinology, Nanjing Medical University, \\ Affiliated Hangzhou Hospital (Hangzhou First People's Hospital), Hangzhou, Zhejiang 310006, P.R. China
}

Received June 7, 2014; Accepted February 27, 2015

DOI: $10.3892 / \mathrm{ol} .2015 .3119$

\begin{abstract}
The aim of the present study was to investigate the prevalence of the $B R A F \mathrm{~V} 600 \mathrm{E}$ mutation in papillary thyroid carcinoma (PTC) patients from eastern coastal China and to determine whether it is correlated with the clinicopathological features of PTCs with or without current Hashimoto thyroiditis (HT). The BRAF V600E mutation status was analyzed in 206 thyroid nodules of 154 patients undergoing thyroidectomy using polymerase chain reaction and bi-directional sequencing. Multivariate analysis was performed to investigate the association of the BRAF V600E mutation with clinicopathological features. Thyroid nodules were classified as PTC, nodular goiter (NG), adenomatoid nodule, adenoma and HT. The BRAF V600E mutation was observed in $61.5 \%$ of PTCs analyzed; it was also detected in one normal tissue adjacent to PTC and one NG. One patient exhibited double mutations in the $B R A F$ gene; the $B R A F$ V600E mutation in the PTC lesion and the BRAF K601E mutation in the contralateral NG lesion. Patients harboring the $B R A F$ V600E mutation had higher thyroid stimulating hormone levels $(2.453 \pm 1.464$ vs. $1.966 \pm 1.296 \mathrm{mIU} / \mathrm{l})$, a reduced occurrence of papillary thyroid microcarcinoma ( $55.0 \mathrm{vs} .88 \%)$, and a higher occurrence of lymph node metastasis (LNM; 42.5 vs. 16.0\%) compared with those with wild-type $B R A F($ all $\mathrm{P}<0.05)$. Binary logistic regression analysis revealed that the $B R A F$ V600E mutation was associated with LNM of PTC (hazard ratio, 5.051; 95\% confidence interval, 1.068-23.893; $\mathrm{P}=0.041$ ). Conversely, no association was identified between the $B R A F$ V600E mutation and HT (38.5 vs. $\left.67.3 \%, \chi^{2}=3.656, \mathrm{P}=0.056\right)$. Thus, in regional PTCs, the BRAF V600E mutation was prevalent, suggesting that it may be an early and phenotypically defining molecular event in PTC, and may represent an independent factor that predicts LNM.
\end{abstract}

Correspondence to: Ms. Lizhen Ma, Department of Endocrinology, Nanjing Medical University, Affiliated Hangzhou Hospital (Hangzhou First People's Hospital), 261 Huansha Road, Hangzhou, Zhejiang 310006, P.R. China

E-mail: malizhen2006@163.com

Key words: BRAF, papillary thyroid carcinoma, prognostic value, Hashimoto thyroiditis

\section{Introduction}

Thyroid carcinoma (TC) is the most common endocrine malignancy (1); according to data presented by the National Comprehensive Cancer Network, it is currently the sixth most common malignancy diagnosed in females (2). The incidence of TC, particularly papillary thyroid carcinoma (PTC), is increasing by $6.2 \%$ per year $(2,3)$.

More than $70 \%$ of PTCs have genetic alterations associated with the mitogen-activated protein kinase pathway (4). $B R A F$ mutations have been commonly observed in all three subtypes of Raf kinase, the first identified and well-characterized downstream cytosolic effector of RAS. Of the greater than $45 B R A F$ mutations that have been identified in human cancer types, approximately $90 \%$ are $\mathrm{T} \rightarrow \mathrm{A}$ transversions in exon 15 at nucleotide 1799 (T1799A), leading to a valine $\rightarrow$ glutamic acid replacement at position 600 (BRAF V600E) (4). In PTC, the prevalence of the $B R A F \mathrm{~V} 600 \mathrm{E}$ mutation varies from 29 to $83 \%$, and occurs mainly in classic PTC (approximately 60\%). In tall-cell variant PTC and PTC-derived anaplastic thyroid cancer, the $B R A F$ V600E mutation was also reported; however, it has rarely been identified in follicular-variant PTC (FV-PTC) and never reported in follicular thyroid carcinoma (FTC) (4).

Numerous studies have demonstrated that the $B R A F$ mutation is the main genetic event in PTC, and is associated with poor prognosis. In rat thyroid PCCL3 cells conditionally expressing oncogenic $B R A F$, increased invasion into Matrigel was observed compared with cells expressing RET/PTC3 in vitro, which was consistent with the biological behavior of human PTC (5). In addition, PTC without the BRAF V600E mutation mainly corresponded to FV-PTC, and maintained a thyroid differentiation expression level close to that of normal tissue and thus had a better prognosis than PTC with the gene alteration (6). Furthermore, the BRAF V600E mutation may induce genetic instability in PTC, facilitating secondary genetic alternation of members of the PI3k/Akt pathway that may induce PTC progression to a more aggressive thyroid cancer (7). However, whether the BRAF V600E mutation represents an independent risk factor for predicting thyroid cancer recurrence or metastasis is a matter of debate. In addition, although PTC is usually accompanied with Hashimoto thyroiditis (HT), which is considered a precancerous lesion of PTC by certain investigators (8), its involvement in the pathogenesis of PTC is controversial. Therefore, this study aimed to investigate 
the prevalence of the BRAF V600E mutation in PTCs with or without current HT in patients from eastern coastal China. Specifically, the association of the BRAF V600E mutation with the clinicopathological features of PTC patients was analyzed. These studies may provide the basis of developing individual diagnostic protocols and treatments for TC patients.

\section{Patients and methods}

Patients. A total of 154 consecutive patients diagnosed with thyroid nodules (206 affected nodules in total) were enrolled from January 2012 to January 2013. All patients (31 males and 123 females, with a mean age of 50.3 years, range 22-76 years) underwent thyroidectomy at the Department of Surgical Oncology at Hangzhou First People's Hospital, (Hangzhou, China). Written informed consent was obtained from all study participants, and the study was approved by the Institutional Ethics Committee of Hangzhou First People's Hospital.

Nodules were classified according to the following diagnostic categories described by the World Health Organization and Diagnostic Histopathology of Tumors (9): PTC (73 patients, 78 nodules), nodular goiter (NG; 65 patients, 95 nodules), adenomatoid nodule (AN; seven nodules), thyroid adenoma (TA; four nodules), and HT (six nodules). HT was diagnosed according to previously described criteria (10): the presence of goiter, increased anti-thyroid antibodies (Tg-Ab, TPO-Ab) by $>50 \%$, or pathological confirmation. Fourteen adjacent normal tissues that were $>1 \mathrm{~cm}$ distance from the PTC were also analyzed as normal controls. Fresh thyroid tissue was quickly frozen at $-80^{\circ} \mathrm{C}$. Of the 206 nodules, 38 were excluded due to insufficient quality of the nucleic acids, poor sequencing signal or improper storage.

Instruments. An Ultrasonic Doppler was purchased from Esaote S.p.A. (Genova, Italy), a multi-image gel imaging system was provided by Bio-Rad Laboratories Inc. (Hercules, CA, USA) and a 2720 Thermal Cycler PCR instrument was obtained from Applied Biosystems (Life technologies Co. Ltd., Foster City, CA, USA).

DNA extraction and direct sequencing. DNA was extracted by fresh-frozen thyroid tissue using a kit (Takara MiniBEST Universal Genomic DNA extraction kit, Ver. 4.0, Code D824A) provided by Bao-Biology Ltd. (Dalian, China) following the manufacturer's recommendations. A 5- $\mu 1$ sample of the DNA template amplified by polymerase chain reaction (PCR) was added in a 50- $\mu 1$ reaction containing $5 \mu \mathrm{l}$ 10X buffer, $4 \mu 1$ dNTPs, $1 \mu 1$ each primer, $0.25 \mu 1$ Taq DNA

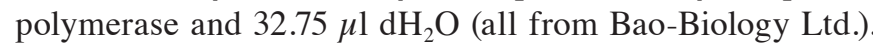
PCR amplification of the $B R A F$ V600E mutation was carried out using the following primers obtained from Huirui Biological Technology Ltd. (Shanghai, China): sense, 5'-ACCTAAACTCTT CATAATGCTTGCT-3' and antisense, 5'-CTGATTTTTGTG AATACTGGGAACT-3'. PCR was carried out with an initial denaturation step at $95^{\circ} \mathrm{C}$ for $5 \mathrm{~min}$; then 30 cycles at $95^{\circ} \mathrm{C}$ for $30 \mathrm{sec}, 52^{\circ} \mathrm{C}$ for $30 \mathrm{sec}$ and $72^{\circ} \mathrm{C}$ for $30 \mathrm{sec}$; and a final extension cycle at $72^{\circ} \mathrm{C}$ for $10 \mathrm{~min}$. Reaction products were visualized on a $1.5 \%$ agarose gel with ethidium bromide, using standard molecular weight DNA as a control.
Bi-directional sequencing was used to detect the presence of the BRAF V600E point mutation by Inhuaiweiji Biological Technology Ltd. (Shanghai, China). DNA sequences were compared with those of the normal BRAF gene exon 15 in the GenBank database using sequence assembly software (Sequencher 4.8; Gene Codes Corporation, Ann Arbor, MI, USA).

Statistical analysis. Statistical analysis was performed using SPSS 13.0 statistical software (SPSS, Inc., Chicago, IL, USA). The association between categorical and continuous variables was evaluated using the two-tailed Fisher's exact test and Student's t-test, respectively. Binary logistic regression analysis was used to assess the correlation between the $B R A F$ V600E mutation and clinicopathological features. $\mathrm{P}<0.05$ was considered to indicate a statistically significant difference.

\section{Results}

Electrophoresis and sequencing results. Representative agarose gel electrophoresis results of the PCR products are shown in Fig. 1, lanes 2-8. Sequencing results are shown in Fig. 2. In one patient, two different $B R A F$ mutations were noted; the $B R A F$ V600E mutation was identified in the PTC lesion, and the BRAF K601E mutation was detected in the contralateral $\mathrm{NG}$ lesion. An $\mathrm{A} \rightarrow \mathrm{G}$ transversion at exon 15 nucleotide 1801 (A1801G) of the BRAF gene resulted in the replacement of lysine with glutamic acid at position 601 (BRAF K601E) as shown in Fig. 3.

Detection of thyroid function and BRAF V600E mutation. Table I shows the levels of T3 and T4 thyroid hormone, free triiodothyronine, free thyroxine and thyroid stimulating hormone (TSH), as well as the prevalence of the BRAF V600E mutation for each group. No significant difference was noted in thyroid function among the groups $(\mathrm{P}>0.05)$. The prevalence of the BRAF V600E mutation in PTCs was $61.5 \%$, which was higher than that observed in patients with benign lesions $\left(\chi^{2}=75.732, \mathrm{P}<0.001\right)$.

Comparisons between 17 NGs and their contralateral PTCs, five cases of bilateral PTCs and 15 cases of bilateral NGs were also conducted. Among the five bilateral PTC patients, two cases had the BRAF V600E mutations in both bilateral PTC lesions, and two cases had wild-type $B R A F$ in both bilateral PTC lesions. The remaining PTC patient carried the $B R A F$ V600E mutation in one PTC lesion, but was negative in the contralateral PTC lesion. Notably, the BRAF V600E mutation was identified in two benign lesions; in normal tissue adjacent to the PTC harboring the BRAF V600E and in NG tissue. The PTC lesion offside to NG tissue carrying the $B R A F$ V600E was also observed to be mutated. In addition, immunohistochemistry analysis revealed that both galectin-3 and HBME-1 were partially expressed in the mutated NG tissue. Conversely, none of the other benign lesions, including 14 bilateral NGs, 38 unilateral NGs, five ANs and three TAs, had the BRAF V600E mutation.

Correlation of the BRAF V600E mutation and HT. After the $\mathrm{NG}, \mathrm{AN}$ and TA groups were merged into a benign tissue (BT) group, this group was compared with the PTC group; 
Table I. Correlation of BRAF V600E mutation and thyroid function in thyroid nodules [n(\%), (mean \pm SD)].

\begin{tabular}{|c|c|c|c|c|c|c|c|c|}
\hline \multirow[b]{2}{*}{ Group } & \multirow[b]{2}{*}{$\mathrm{n}$} & \multicolumn{2}{|c|}{$B R A F$ V600E mutation } & \multirow[b]{2}{*}{$\mathrm{T} 3(\mu \mathrm{g} / \mathrm{l})$} & \multirow[b]{2}{*}{$\mathrm{T} 4(\mu \mathrm{g} / \mathrm{l})$} & \multirow[b]{2}{*}{ FT3 (pmol/l) } & \multirow[b]{2}{*}{ FT4 (pmol/l) } & \multirow[b]{2}{*}{$\mathrm{TSH}(\mathrm{mIU} / \mathrm{l})$} \\
\hline & & - & + & & & & & \\
\hline NT & 14 & 13 (92.9) & $1(7.1)$ & $1.04 \pm 0.26$ & $97.58 \pm 22.02$ & $4.66 \pm 0.43$ & $16.02 \pm 2.44$ & $2.15 \pm 1.21$ \\
\hline $\mathrm{NG}$ & 77 & 76 (98.7) & $1(1.3)$ & $1.07 \pm 0.40$ & $93.29 \pm 17.05$ & $4.75 \pm 0.46$ & $16.53 \pm 7.79$ & $2.02 \pm 1.55$ \\
\hline $\mathrm{AN}$ & 5 & $5(100.0)$ & $0(0.0)$ & $1.08 \pm 0.29$ & $86.45 \pm 26.00$ & $4.51 \pm 0.93$ & $13.29 \pm 3.79$ & $2.65 \pm 2.87$ \\
\hline TA & 3 & $3(100.0)$ & $0(0.0)$ & $1.16 \pm 0.40$ & $106.83 \pm 29.53$ & $4.78 \pm 0.59$ & $15.80 \pm 1.07$ & $1.65 \pm 0.92$ \\
\hline $\mathrm{TC}$ & 65 & $25(36.9)$ & $40(61.5)^{\mathrm{a}}$ & $1.07 \pm 0.56$ & $90.72 \pm 21.78$ & $4.59 \pm 0.73$ & $19.00 \pm 15.56$ & $2.34 \pm 1.37$ \\
\hline HT & 4 & $4(100.0)$ & $0(0.0)$ & $1.61 \pm 1.66$ & $60.87 \pm 36.17$ & $4.31 \pm 0.82$ & $13.19 \pm 7.64$ & $3.04 \pm 1.71$ \\
\hline
\end{tabular}

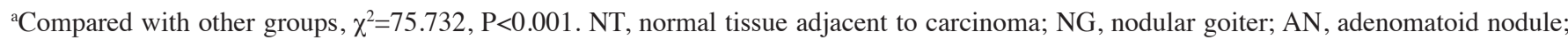
TA, thyroid adenoma; TC, thyroid carcinoma; HT, Hashimoto thyroiditis; -, negative; +, positive; FT3, free triiodothyronine; FT4, free thyroxine; TSH, thyroid stimulating hormone.

Table II. Prevalence of BRAF V600E mutation in thyroid nodules in relation to Hashimoto thyroiditis [n(\%)].

\begin{tabular}{|c|c|c|c|c|c|c|}
\hline \multirow[b]{2}{*}{ Group } & \multirow[b]{2}{*}{ HT } & \multirow[b]{2}{*}{$\mathrm{n}$} & \multicolumn{2}{|c|}{$B R A F$ V600E mutation } & \multirow[b]{2}{*}{$\chi^{2}$} & \multirow[b]{2}{*}{ P-value } \\
\hline & & & - & + & & \\
\hline \multirow[t]{2}{*}{ BT } & - & 71 & $70(98.6)$ & $1(1.4)$ & 0.256 & 0.613 \\
\hline & + & 18 & $18(100.0)$ & $0(0.0)$ & & \\
\hline \multirow[t]{2}{*}{ РTC } & - & 52 & $17(32.7)$ & $35(67.3)$ & 3.656 & 0.056 \\
\hline & + & 13 & $8(61.5)$ & $5(38.5)$ & & \\
\hline
\end{tabular}

BT, benign tissue; PTC, papillary thyroid carcinoma, HT, Hashimoto thyroiditis; -, negative; +, positive.

Table III. Correlation of BRAF V600E mutation and clinicopathological characteristics in thyroid nodules [n(\%), (mean \pm SD)].

\begin{tabular}{|c|c|c|c|c|c|c|c|c|}
\hline & \multicolumn{2}{|c|}{ Gender } & \multirow[b]{2}{*}{ Age (years) } & \multirow[b]{2}{*}{ TSH (mIU/l) } & \multicolumn{2}{|c|}{ PTMC } & \multicolumn{2}{|c|}{ Lymph node metastasis } \\
\hline & Male & Female & & & - & + & - & + \\
\hline$B R A F$ V600E - & $21(16.7)$ & $105(83.3)$ & $51.1 \pm 10.1$ & $1.966 \pm 1.296$ & $3(12.0)$ & $22(88.0)$ & $21(84.0)$ & $4(16.0)$ \\
\hline$B R A F \mathrm{~V} 600 \mathrm{E}+$ & $10(23.8)$ & $32(76.2)$ & $49.0 \pm 11.1$ & $2.453 \pm 1.464$ & $18(45.0)$ & $22(55.0)$ & $23(57.5)$ & $17(42.5)$ \\
\hline Total & $31(18.5)$ & $137(81.5)$ & $50.6 \pm 10.4$ & $2.198 \pm 1.514$ & $21(32.3)$ & $44(67.7)$ & $44(67.7)$ & $21(32.3)$ \\
\hline & \multicolumn{2}{|c|}{$\chi^{2}=1.068$} & $\mathrm{t}=1.134$ & $\mathrm{t}=2.019$ & \multicolumn{2}{|c|}{$\chi^{2}=7.661$} & \multicolumn{2}{|c|}{$\chi^{2}=4.940$} \\
\hline P-value & \multicolumn{2}{|c|}{0.301} & 0.259 & 0.045 & \multicolumn{2}{|c|}{0.006} & \multicolumn{2}{|c|}{0.026} \\
\hline
\end{tabular}

PTMC, papillary thyroid microcarcinoma; TSH, thyroid stimulating hormone; -, negative; +, positive.

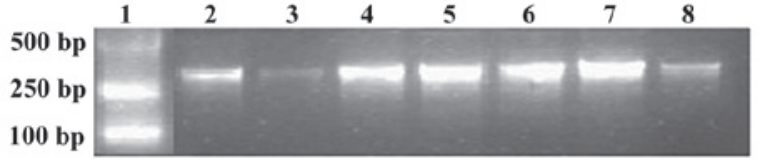

Figure 1. Agarose gel (1.5\%) electrophoresis for thyroid tissue polymerase chain reaction products. Lane 1, DL2000 DNA marker (Dingguo Biology Ltd., Beijing, China). Lanes 2-8, representative positive cases.

there was no significant difference in the prevalence of patients with concurrent HT between the two groups (20.0 vs. $20.2 \%$, $\chi^{2}=0.001, \mathrm{P}=0.973$; Table II). The BRAF V600E mutation was detected mainly in PTCs $\left(\chi^{2}=70.186, \mathrm{P}<0.001\right)$. Comparative analysis using layered HT from BT and PTC indicated that the prevalence of the $B R A F$ V600E mutation was lower in PTCs accompanied with HT than in those without HT (38.5 vs. 67.3\%), but it was not statistically significant $\left(\chi^{2}=3.656, \mathrm{P}=0.056\right)$.

Correlation of the BRAF V600E mutation with clinicopathological features. As shown in Table III, no significant difference in gender $\left(22.8\right.$ vs. $\left.16.7 \%, \chi^{2}=1.068, \mathrm{P}=0.301\right)$ or age $(49.0 \pm 11.1$ vs. $51.1 \pm 10.1$ years, $\mathrm{t}=1.134, \mathrm{P}=0.259)$ was noted between patients with the mutated and non-mutated nodules. However, patients harboring the BRAF V600E 
Table IV. Associations between clinicopathological features and lymph node metastasis of papillary thyroid carcinoma.

\begin{tabular}{lccc}
\hline Clinical features & P-value & HR & 95\% CI \\
\hline Gender & 0.016 & 0.147 & $0.031-0.697$ \\
Age, years & 0.279 & & \\
TSH level & 0.374 & & \\
PTMC & 0.181 & & \\
HT & 0.121 & 5.051 & $1.068-23.893$ \\
BRAF mutation & 0.041 &
\end{tabular}

TSH, thyroid stimulating hormone; PTMC, papillary thyroid microcarcinoma; HT, Hashimoto thyroiditis; HR, hazard ratio; CI, confidence interval.
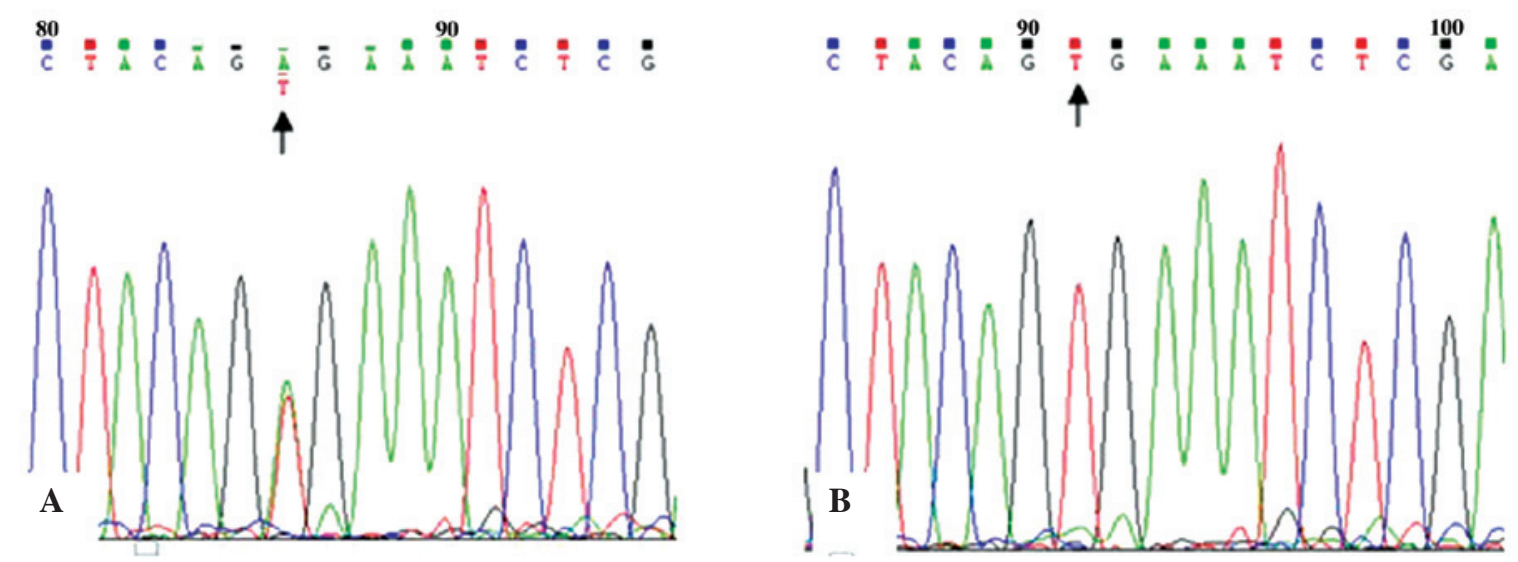

Figure 2. (A) Representative sequencing traces from one of the positive replicates indicating the BRAF V600E heterozygous mutation, a $\mathrm{T}$ to $\mathrm{A}$ transversion at exon 15 nucleotide 1799 (T1799A) of the BRAF gene; (B) the wild type.

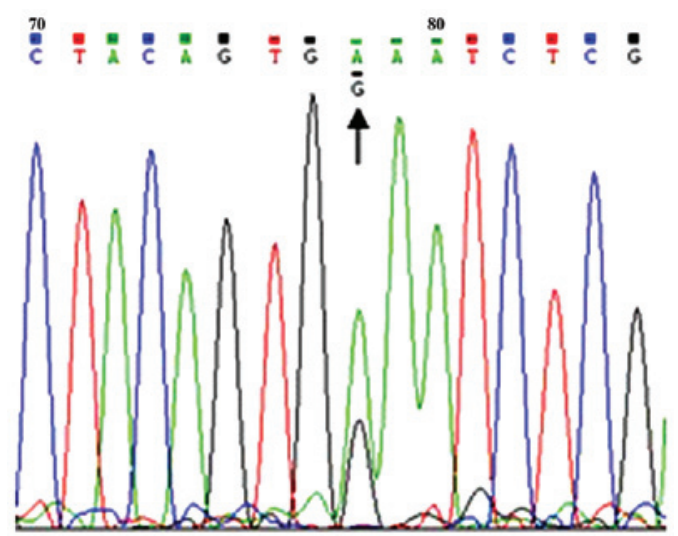

Figure 3. Sequencing traces from one patient indicating the $B R A F \mathrm{~K} 601 \mathrm{E}$ heterozygous mutation, an A to $\mathrm{G}$ transversion at exon 15 nucleotide 1801 (A1801G) of the $B R A F$ gene.

mutation had higher TSH levels compared with the patients with wild-type $B R A F(2.453 \pm 1.464$ vs. $1.966 \pm 1.296 \mathrm{mIU} / \mathrm{l}$, respectively; $\mathrm{t}=2.019 ; \mathrm{P}=0.045)$. Among the PTCs, $44(67.7 \%)$ were papillary thyroid microcarcinomas (PTMCs) with a diameter of $1 \mathrm{~cm}$ or less. Compared with non-mutated PTCs, PTCs harboring the BRAF V600E mutation were less likely to be a PTMC (55.0 vs. $88 \%, \mathrm{P}<0.05)$, and they had a higher occurrence of lymph node metastasis (LNM; 42.5 vs. $16.0 \%$,
$\mathrm{P}<0.05)$. Similarly, PTMC tumors harboring the $B R A F$ V600E mutation had a higher incidence of LNM, although the difference was not significant (36.4 vs. $13.6 \%, \chi^{2}=3.030$, $\mathrm{P}=0.162$ ).

Next, correlation analysis of the BRAF V600E mutation and clinicopathological features was conducted. Binary logistic regression analysis revealed that LNM of PTC was associated with the BRAF V600E mutation, gender and PTMC, but not with age, TSH or HT (Table IV). In addition, the BRAF V600E mutation [hazard ratio (HR), 5.051; 95\% confidence interval $(\mathrm{CI}), 1.068-23.893 ; \mathrm{P}=0.041)$ and female gender (HR, 0.147; 95\% CI, 0.031-0.697; $\mathrm{P}=0.016)$ were independent factors that predicted LNM.

\section{Discussion}

The prevalence of the BRAF V600E mutation in PTCs ranges from 29 to $83 \%$ and is reported only in malignant thyroid tumors $(4,11,12)$; similarly, the incidence of the $B R A F$ V600E mutation was $61.5 \%$ in PTC patients from China's eastern coast in this study. All of the BRAF V600E mutations identified in these PTCs were heterozygous mutations. Similar to the results of previous studies, most of the benign lesions, including 14 bilateral NGs, 38 unilateral NGs, five ANs and three TAs, carried the wild-type $B R A F$ gene. In contrast to other studies that failed to identify $B R A F$ mutations in benign 
thyroid disease, two benign tissues carried the $B R A F$ V600E mutation in the present study. In the first case, the mutation was detected in normal tissue adjacent to PTC that also carried the $B R A F$ V600E mutation, suggesting the presence of infiltrative growth. In the second case, the $B R A F$ V600E mutation was detected in the NG tissue, on the contralateral PTC lesion also carrying the mutation, suggesting that this mutation may represent an early event in PTC progression. Moreover, immunohistochemistry analysis of galectin-3 and HBME-1 expression in the $B R A F$ V600E-positive NG revealed that both were weakly expressed. Combined immunohistochemistry for galectin-3 and HBME-1 was useful for the differentiation of benign and malignant thyroid tumors (13); therefore, the mutation in the NG tissue may be indicative of a trend toward malignant biological progression. In summary, the $B R A F$ V600E mutation may represent a critical step in tumor progression.

Another notable finding in the present study was that one patient exhibited double mutations in the $B R A F$ gene; the $B R A F$ V600E mutation was identified in the PTC lesion, and the BRAF K601E mutation was identified in the contralateral $\mathrm{NG}$ lesion. An $\mathrm{A} \rightarrow \mathrm{G}$ transversion at exon 15 nucleotide 1801 (A1801G) of the $B R A F$ gene resulted in the replacement of lysine with glutamic acid at position 601 (BRAF K601E), which was also a heterozygous mutation in this patient. Trovisco et al (14) first reported the $B R A F \mathrm{~K} 601 \mathrm{E}$ mutation in a PTC patient in 2005, and 18 additional cases have been subsequently described (15-24). Meta-analysis demonstrated that the $B R A F$ K601E mutation was predominantly identified in FV-PTC, followed by FTC and PTC, and only one case of TA had the $B R A F$ K601E mutation. Although it may affect the PI3k/Akt pathway, this mutation exhibited relatively inactive biological characteristics in the pathogenesis of TC compared with the $B R A F$ V600E mutation (15-24). Moreover, in multifocal papillary thyroid carcinoma (mPTC), individual tumor foci may be identical and are frequently composed of various histological types, and individual tumor foci also harbor different mutations. For example, Kim et al (23) described a case of mPTC consisting of one FV-PTC harboring the BRAF K601E mutation and three conventional foci harboring the $B R A F \mathrm{~V} 600 \mathrm{E}$ mutation. Similarly, we present a patient with both the $B R A F$ V600E and $B R A F$ K601E mutations although the BRAF K601E mutation was detected in a NG. To our knowledge, this is the first study of a $B R A F$ K601E mutation in NG tissue. We suspect that the BRAF K601E mutation in the NG tissue may be an indication of oncogene activation that is believed to take place early in the course of tumorigenesis. However, determining the exact mechanism requires further research.

Numerous studies have demonstrated an association between the BRAF V600E mutation with aggressive clinicopathological characteristics of PTC, including extrathyroidal invasion, LNM, advanced tumor-node-metastasis stage, loss of radioiodine avidity and disease recurrence (5-7). BRAF mutation presents a low positive predictive value $(28 \%)$ and a high negative predictive value $(87 \%)$ for PTC recurrence, suggesting that $B R A F$ mutational status in clinical management of PTC should be used with caution. However, it is still controversial to use $B R A F$ mutation as an independent predictive risk factor for PTC due to the fact that the $B R A F \mathrm{~V} 600 \mathrm{E}$ mutation is identified in approximately half of PTCs, among which less than $10-15 \%$ of the tumors exhibit aggressive behavior (25). In accordance with previous studies, a higher rate of LNM was noted in BRAF V600E-positive PTCs than in negative ones at the time of surgery in this study. In addition, binary logistic regression analysis indicated that this mutation was an independent predictive factor of LNM in PTC patients. The proportion of PTMC was lower in PTCs with the $B R A F$ V600E mutation than in those with the wild-type gene. Taken together, this mutation may promote tumor growth and LNM; therefore, the $B R A F \mathrm{~V} 600 \mathrm{E}$ mutation was correlated with a more aggressive behavior of PTC.

Even though tumor-related mortality is as low as $0.5 \%$ for PTMC, microcarcinoma harboring the BRAF V600E mutation is also associated with features predictive of a high risk of recurrence and metastasis (26-28). In our study, $67.7 \%$ (44/65) of PTCs were PTMCs, among which 50\% (22/44) of the patients had the $B R A F \mathrm{~V} 600 \mathrm{E}$ mutation. Consistent with previous studies, the presence of the $B R A F$ V600E mutation in PTMC further suggests that the mutation is an early event in thyroid carcinogenesis. Similarly, patients with PTMCs harboring the BRAF V600E mutation had a higher incidence of LNM although this was not significant $(\mathrm{P}=0.162)$. However, we expect that this correlation will be confirmed in further studies with larger sample sizes.

A number of studies $(29,30)$ have reported a weak association between serum TSH concentration and thyroid cancer, with certain investigators suggesting that higher preoperative TSH serum concentration may be associated with advanced tumor stage and poor prognosis (29); however, the correlation between TSH and PTC remains controversial. Although TSH suppressive therapy is a well-known adjuvant therapy to prevent recurrence in differentiated thyroid cancer, no significant difference in TSH level was identified among different pathological types in the present study, which may be due to the insufficient sample size. In the present study, compared with tumors with wild-type $B R A F$, patients with PTCs harboring the BRAF V600E mutation had higher TSH levels. Whether the mutation is involved in the activation of TSH receptor remains unclear and requires further analysis.

PTC with HT is frequently observed; however, the association between HT and PTC is controversial. When compared with benign lesions, there was no significant difference in the rate of PTCs with concurrent HT; therefore, the correlation between HT and TCs remains unclear. However, Kim et al (30) reported that in Korean patients with PTC, the $B R A F$ V600E mutation was associated with a low frequency of background HT and a high frequency of LNM. Similarly, PTCs accompanied with HT had a lower frequency of the $B R A F$ V600E mutation in our study, although this was not significant $(\mathrm{P}=0.056)$. However, additional analysis in an expanded sample size may yield significant differences.

In conclusion, in PTC patients from China's eastern coast, the BRAF V600E mutation was prevalent (61.5\%). Similar to findings in previous studies, our results supported the notion that the mutation was an early and phenotypically defining molecular event in PTC, associated with features predictive of a high risk of LNM even in microcarcinomas. In addition, one patient in our study exhibited two mutations in the $B R A F$ gene; $B R A F$ V600E and $B R A F$ K601E, with the latter identified in NG tissue. 


\section{Acknowledgements}

This study was supported by grants from the Medical Science Research Foundation of Zhejiang Province (2010KYA161) and the Key Project of Scientific and Technological Innovation in Hangzhou (20131813A08).

\section{References}

1. Davies L and Welch HG: Increasing incidence of thyroid cancer in the United States, JAMA 295: 2164-2167, 2006.

2. Tuttle RM, Ball DW, Byrd D, et al: Thyroid carcinoma. J Natl Compr Canc Netw 8: 1228-1274, 2010.

3. Veiga LH, Neta G, Aschebrook-Kilfoy B, Ron E and Devesa SS: Thyroid cancer incidence patterns in Sao Paulo, Brazil and the US SEER Program. Thyroid 23: 748-757, 2013.

4. Tang KT and Lee CH: BRAF mutation in papillary thyroid carcinoma: pathogenic role and clinical implications. J Chin Med Assoc 73: 113-128, 2010.

5. Mesa C Jr, Mirza M, Mitsutake N, et al: Conditional activation of RET/PTC3 and BRAFV600E in thyroid cells is associated with gene expression profiles that predict a preferential role of BRAF in extracellular matrix remodeling. Cancer Res 66: 6521-6529, 2006.

6. Durand S, Ferraro-Peyret C, Joufre M, Chave A, Borson-Chazot F, Selmi-Ruby S and Rousset B: Molecular characteristics of papillary thyroid carcinomas without BRAF mutation or RET/PTC rearrangement: relationship with clinico-pathological features. Endocr Relat Cancer 16: 467-481, 2009.

7. Xing M: Genetic alterations in the phosphatidylinositol-3 kinase/ Akt pathway in thyroid cancer. Thyroid 20: 697-706, 2010.

8. Konturek A, Barczyński M, Wierzchowski W, Stopa M and Nowak W: Coexistence of papillary thyroid cancer with Hashimoto thyroiditis. Langenbecks Arch Surg 398: 389-394, 2013.

9. Zhou GY, Liu HQ and Zhang QH: Thyroid and parathyroid gland tumors. In: Diagnostic Histopathology of Tumors. Fletcher CD (ed). 1st edition. Shandong Science and Technology Press, Jinan, pp599, 2001.

10. Liao EY and Chao CS. Chronic lymphocytic thyroiditis. In: Endocrinology. Zhang H, Dai RC, Liao EY (eds). 1st edition. People's Medical Publisher, Beijing, pp715, 2001.

11. Gouveia C, Can NT, Bostrom A, Grenert JP, van Zante A and Orloff LA: Lack of association of BRAF mutation with negative prognostic indicators in papillary thyroid carcinoma: the University of California, San Francisco, experience. JAMA Otolaryngol Head Neck Surg 139: 1164-1170, 2013.

12. Witt RL, Ferris RL, Pribitkin EA, Sherman SI, Steward DL and Nikiforov YE: Diagnosis and management of differentiated thyroid cancer using molecular biology. Laryngoscope 123: 1059-1064, 2013.

13. Yu LY, Zhang C, Xu RJ, Zhou H and Ma LZ: Diagnostic value of TPO, CK-19, Gal-3 and HBME-1 in fine needle aspiration cytological specimens for thyroid carcinoma with Hashimoto's disease. Zhejiang Medical 32: 1007-1010, 1013, 2010.

14. Trovisco V, Soares P, Soares R, Magalhães J, Sá-Couto P and Sobrinho-Simões M: A new BRAF gene mutation detected in a case of a solid variant of papillary thyroid carcinoma. Hum Pathol 36: 694-697, 2005.
15. Schmidt J, Derr V, Heinrich MC, Crum CP, Fletcher JA, Corless CL and Nosé V: BRAF in papillary thyroid carcinoma of ovary (struma ovarii). Am J Surg Pathol 31: 1337-1343, 2007.

16. Lupi C, Giannini R, Ugolini C, et al: Association of BRAF V600E mutation with poor clinicopathological outcomes in 500 consecutive cases of papillary thyroid carcinoma. J Clin Endocrinol Metab 92: 4085-4090, 2007.

17. Frau DV, Lai ML, Caria P, et al: Trisomy 17 as a marker for a subset of noninvasive thyroid nodules with focal features of papillary carcinoma: cytogenetic and molecular analysis of 62 cases and correlation with histological findings. J Clin Endocrinol Metab 93: 177-181, 2008.

18. Wolff EF, Hughes M, Merino MJ, Reynolds JC, Davis JL, Cochran CS and Celi FS: Expression of benign and malignant thyroid tissue in ovarian teratomas and the importance of multimodal management as illustrated by a BRAF-positive follicular variant of papillary thyroid cancer. Thyroid 20: 981-987, 2010.

19. Pennelli G, Vianello F, Barollo S, et al: BRAF (K601E) mutation in a patient with a follicular thyroid carcinoma. Thyroid 21: 1393-1396, 2011

20. Ohori NP, Singhal R, Nikiforova MN, et al: BRAF mutation detection in indeterminate thyroid cytology specimens: underlying cytologic, molecular and pathologic characteristics of papillary thyroid carcinoma. Cancer Cytopathol 121: 197-205, 2013

21. Schulten HJ, Salama S, Al-Mansouri Z, et al: BRAF mutations in thyroid tumors from an ethnically diverse group. Hered Cancer Clin Pract 10: 10-11, 2012.

22. Park JY, Kim WY, Hwang TS, et al: BRAF and RAS mutations in follicular variants of papillary thyroid carcinoma. Endocr Pathol 24: 69-76, 2013.

23. Kim WY, Ko YS and Hwang TS: A case of multifocal papillary thyroid carcinoma consisting of one encapsulated follicular variant with BRAF K601E mutation and three conventional types with BRAF V600E mutation. Korean J Pathol 47: 293-298, 2013.

24. Barollo S, Pezzani R, Cristiani A, et al: Prevalence, tumorigenic role and biochemical implications of rare BRAF alterations. Thyroid 24: 809-819, 2014.

25. Soares P,Celestino R,Melo M,Fonseca Eand Sobrinho-Simões M: Prognostic biomarkers in thyroid cancer. Virchows Archiv 464: 333-346, 2014.

26. RotiE, degli UbertiEC,Bondanelli Mand Braverman LE: Thyroid papillary microcarcinoma: a descriptive and meta-analysis study. Eur J Endocrinol 159: 659-673, 2008.

27. Lee X, Gao M, Ji Y, et al: Analysis of differential BRAF (V600E) mutational status in high aggressive papillary thyroid microcarcinoma. Ann Surg Oncol 16: 240-245, 2009.

28. Virk RK, Van Dyke AL, Finkelstein A, et al: BRAFV600E mutation in papillary thyroid microcarcinoma: a genotype-phenotype correlation. Mod Pathol 26: 62-70, 2013.

29. Kim D and Park JW: Clinical implications of preoperative thyrotropin serum concentrations in patients who underwent thyroidectomy for nonfunctioning nodule(s). J Korean Surg Soc 85: 15-19, 2013.

30. Kim SK, Song KH, Lim SD, Lim YC, Yoo YB, Kim JS and Hwang TS: Clinical and pathological features and the BRAF (V600E) mutation in patients with papillary thyroid carcinoma with and without concurrent Hashimoto thyroiditis. Thyroid 19: 137-141, 2009. 